\title{
A Validated Stability Indicating LC Method for Amlexanox in Bulk Drugs
}

\author{
Bethanabhatla Syama Sundar ${ }^{*}$, Mohammed Nazeerunnisa \\ ${ }^{1}$ Department of Chemistry, Acharya Nagarjuna University, Nagarjuna Nagar, Guntur, India \\ E-mail: profbsyamsundar@yahoo.co.in \\ Received March 2, 2011; revised May 10, 2011; accepted July 4, 2011
}

\begin{abstract}
A novel and sensitive stability indicating RP-HPLC method has been developed for the quantitative determination of amlexanox in bulk drugs. The separation was accomplished on $\mathrm{C}_{18}$ column using $10 \mathrm{mM}$ ammonium dihydrogen orthophosphate (pH adjusted to 4.8 by using ortho phosphoric acid) and methanol (30:70 $v / v)$ as mobile phase in an isocratic elution mode at a flow rate of $1.0 \mathrm{~mL} \mathrm{~min}^{-1}$. The eluents were monitored by PDA detector at $245 \mathrm{~nm}$. The drug was subjected to stress conditions of hydrolysis, oxidation, photolysis and thermal degradation. Significant degradation was found under basic, acidic stress and UV light. The resolution $\left(R_{s}\right)$ between amlexanox and its degradation products was found to be greater than 2.5. Regression analysis shows correlation coefficient greater than 0.999 for amlexanox. The inter and intraday precision values for amlexanox were found to be within 1.0\% RSD. The method has shown good and consistent recoveries for amlexanox in bulk drugs $(98.86 \%$ - 101.05\%). The developed method was validated with respect to linearity, accuracy, precision and robustness.
\end{abstract}

Keywords: RP-HPLC, Amlexanox, Degradation, Validation, Stability indicating

\section{Introduction}

Amlexanox is a novel anti-inflammatory and anti-allergic agent that has been evaluated for the treatment of recurrent aphthous ulcers (RAU) and is currently the only clinically proven product approved by the US FDA for the treatment of aphthous ulcers. The chemical name of amlexanox is 2-acid amino-7-isopropyl-5-oxo-5Hchromeno [2,3-b] pyridine-3-carboxylic acid (Figure 1). RAU is the most prevalent oral mucosal disease in humans. Prior to amlexanox available treatment was largely symptomatic, with patient management being either entirely empiric or based on clinicians' perception of the cause of the ulcers. Amlexanox effectively treats aphthous ulcers by accelerating healing of ulcer and by accelerating complete resolution of pain. Amlexanox is commercially available as $5 \%$ oral paste and as biodegradable muco-adhesive disc. Amlexanox potently inhibits the release of histamine and leukotrienes from mast cells, basophils and neutrophils under invitro settings, possibly through increasing intracellular cyclic AMP content in inflammatory cells, a membrane-stabilizing effect or inhibition of calcium influx [1-5].

A few HPLC methods were reported in the literature for the analysis of amlexanox which includes like validation of HPLC-FL assay for the determination of amlexanox in human serum [6], RP-HPLC method for three distinct anti-allergic drugs to bind the proteins: Amlexanox, cromolyn and tranilast [7]. Study on pharmacokinetics and demonstration of clinical safety for amlexanox $5 \%$ oral paste $[8,9]$, stable viscous liquid formulations of amlexanox for the prevention and treatment of mucosal diseases and disorders [10].

Extensive literature survey reveals that there is no stability-indicating LC method for determination and for the quantitative estimation of amlexanox in bulk drugs. An ideal stability indicating chromatographic method for estimation of any drug should be able to resolve from

Amlexanox:

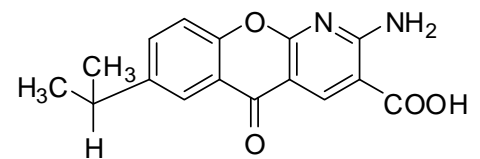

IUPAC Name: 2- acid amino-7-isopropyl-5-oxo-5H-chromeno[2,3-b] pyridine-3-carboxylic acid

Molecular Formula: $\mathrm{C}_{16} \mathrm{H}_{14} \mathrm{~N}_{2} \mathrm{O}_{4}$

Molecular weight: $298 \mathrm{~g} / \mathrm{mol}$

Figure 1. Chemical Structures and labels of Amlexanox. 
degradation products. The present drug stability test guideline Q1A (R2) issued by International Conference on Harmonization (ICH) suggests that stress studies should be carried out on a drug to establish its inherent stability characteristics, leading to the separation of degradation products and hence supporting the suitability of the proposed analytical procedures. It also requires being stability indicating besides validated fully. [11-13].

Hence, an attempt has been made to develop an accurate, rapid, specific and reproducible method for the determination of amlexanox in bulk drug samples along with method validation as per ICH norms. The stability tests were also performed on both drug substances as per ICH norms.

\section{Experimental}

\subsection{Chemicals}

Amlexanox and its standard were obtained from Star Lab Tech Pharmaceuticals, Hyderabad, India. HPLC grade methanol, ortho phosphoric acid and analytical reagent grade ammonium dihydrogen phosphate were of Merck, Darmstadt, Germany. High purity water was prepared by using Millipore Milli-Q plus water purification system. All samples and impurity used in this study were of greater than $99.6 \%$ purity.

\subsection{Equipment}

The LC system used for method development, forced degradation studies and method validation were Waters 2695 binary pump plus auto sampler and a 2996 photo diode array detector (Waters Corporation, MA, USA). The output signal was monitored and processed using Empower software on Pentium computer (Digital equipment Co) and Agilent 1200 series DAD (diode array detector) with Empower soft ware. Photo stability studies were carried out in a photo stability chamber (MACK Pharmatech). Thermal stability studies were performed in a dry air oven (Mack Pharmatech, Hyderabad, India).

\subsection{Chromatography}

The chromatographic column used was Intersil ODS-4, $(4.6 \times 250) \mathrm{mm} ; 5 \mu \mathrm{m}$. The mobile phase consists of a mixture of buffer and methanol in the ratio of 30:70 v/v. Buffer consists of $10 \mathrm{mM}$ ammonium dihydrogen orthophosphate, $\mathrm{pH}$ adjusted to 4.8 using ortho phosphoric acid. The column temperature was maintained at $25^{\circ} \mathrm{C}$ and the detection was monitored at a wavelength of 245 $\mathrm{nm}$. The injection volume was $10 \mu \mathrm{L}$. Mobile phase being used as diluent.

\subsection{Preparation of Standard Solutions}

About $12 \mathrm{mg}$ portion of amlexanox standard was weighed into standard $100 \mathrm{ml}$ volumetric flask, dissolved in and diluted to volume with diluent. $5 \mathrm{ml}$ of above solution was transferred into a $10 \mathrm{ml}$ volumetric flask and diluted to volume with diluent.

\subsection{Preparation of Stress/System Suitability Samples}

About $50 \mathrm{mg}$ of amlexanox sample was taken into a 50 $\mathrm{mL}$ volumetric flask and made up to the mark with $0.1 \mathrm{~N}$ sodium hydroxide and refluxed at $100^{\circ} \mathrm{C}$ for $1 \mathrm{hr}$. An aliquot of $0.6 \mathrm{~mL}$ was pipette out into a $10 \mathrm{~mL}$ volumetric flask, neutralized with $1 \mathrm{~N} \mathrm{HCl}$ and made up to the volume with mobile phase.

\subsection{Specificity/ Application of Stress (Forced Degradation Study)}

Specificity is the ability of the method to measure the analyte response in the presence of its potential impurities [14] which can help to identify the likely degradation products and establish its pathways and the intrinsic stability of the molecule and validate the stability indicating power of the analytical procedures used

The specificity of the developed LC method for amlexanox was determined in the presence of its degradation products. Forced degradation studies were also performed on amlexanox to understand the stability indicating property and specificity of the proposed method. The stress conditions employed for degradation study includes light (carried out as per ICH Q1B), heat $\left(105^{\circ} \mathrm{C}\right.$ for $48 \mathrm{hrs})$, acid hydrolysis $(1 \mathrm{~N} \mathrm{HCl})$, base hydrolysis $(0.1 \mathrm{~N} \mathrm{NaOH})$, water hydrolysis and oxidation $\left(5 \% \mathrm{H}_{2} \mathrm{O}_{2}\right)$. Amlexanox is exposed to $200 \mathrm{w} / \mathrm{hm}^{2} \mathrm{UV}$ light in solution and solid states. Amlexanox is exposed to 1.2 million flux hours fluorescent light in solution and solid state. Amlexanox solution is exposed to ultrasonic bath for $1 \mathrm{hr}$ at $25^{\circ} \mathrm{C}$. Peak purity of stressed samples of amlexanox was checked by using 2996 Photo diode array detector of Waters (PDA).

Assays were carried out for the stress samples against a qualified reference standard. The mass balance (\% assay $+\%$ of impurities $+\%$ of degradation products) was calculated for all of the samples.

\subsection{Analytical Method Validation}

The developed chromatographic method was validated for linearity, range, precision, accuracy, sensitivity, and robustness. 


\subsubsection{Precision}

The precision of the amlexanox method was checked by injecting six individual preparations of $\left(60 \mu \mathrm{gL}^{-1}\right)$ in triplicate (intraday) on the same day. The \%RSD area of amlexanox was calculated. Precision study was also determined by performing the same procedures on three different days (inter-day precision).

The intermediate precision (ruggedness) of the method was also evaluated by different experimenter, different column and different instrument in the same laboratory.

Assay method precision was evaluated by carrying out six independent determinations of test sample of amlexanox against qualified reference standard. The \%RSD values of six determinations obtained were calculated. The intermediate precision of the assay method was evaluated by different experimenter and by using different instrument from the same laboratory.

\subsubsection{Sensitivity}

Sensitivity was determined by establishing the Limit of detection (LOD) and Limit of quantitation (LOQ) for amlexanox estimated at a signal-to-noise ratio of 3:1 and 10:1 respectively by injecting a series of dilute solutions with known concentration. The precision study was also carried out at the LOQ level by injecting six individual preparations of amlexanox and the values of \%RSD calculated for the areas of the amlexanox.

\subsubsection{Linearity and Range}

To establish linearity of the assay method, calibration solutions were prepared from stock solution at five concentration levels from $80 \%$ to $120 \%$ of assay analyte concentrations (48, 54, 60, 66 and $72 \mu \mathrm{g} \mathrm{mL} \mathrm{L}^{-1}$ ). Linearity was checked for three consecutive days in the same concentration range. Upper and lower levels of range were also established.

\subsubsection{Accuracy}

The accuracy of the assay method was evaluated in triplicate at three concentration levels, i.e. 48, 60 and $72 \mu \mathrm{g}$ $\mathrm{mL}^{-1}$ in bulk drugs. For each concentration, three sets were prepared and injected in triplicate. The percentage of recovery was calculated at each level.

\subsubsection{Robustness}

To determine the robustness of the developed method, experimental conditions were deliberately changed and the resolution $\left(R_{S}\right)$ between amlexanox and its degradant in basic condition was evaluated. The effect of flow rate on the resolution was studied with 0.8 and $1.2 \mathrm{~mL} \cdot \mathrm{min}^{-1}$ while the optimized flow rate of the mobile phase was $1.0 \mathrm{~mL} \cdot \mathrm{min}^{-1}$. The effect of column temperature on resolution was studied at $20^{\circ} \mathrm{C}$ and $30^{\circ} \mathrm{C}$ instead of $25^{\circ} \mathrm{C}$.
The effect of $\mathrm{pH}$ on resolution of impurity was studied by varying $\pm 0.1 \mathrm{pH}$ units (i.e. buffer $\mathrm{pH}$ altered from 4.8 to 4.7 and 4.9 ). In the all above varied conditions, the components of the mobile phase were held constant.

\subsubsection{Solution Stability and Mobile Phase Stability}

The solution stability of amlexanox in the assay method was carried out by leaving the test solutions of samples in tightly capped volumetric flasks at room temperature for $48 \mathrm{hrs}$. The same sample solutions were assayed at 0 hrs, 18 hrs, 24 hrs, 42 hrs and 48 hrs against freshly prepared standard solutions. The mobile phase stability was also carried out by determining the freshly prepared sample against freshly prepared reference standard solutions at 0 hrs, 18 hrs, 24 hrs, 42 hrs and 48 hrs. The \%RSD of assay of amlexanox was calculated for the study period during mobile phase and solution stability experiments.

\section{Results and Discussion}

\subsection{Method Development and Optimization}

The objective of the present work was to develop a stability-indicating liquid chromatographic analytical method for the determination of amlexanox in bulk drugs. Amlexanox standard was used during the method development. To develop a rugged and suitable LC method for the amlexanox, different mobile phases and stationary phases were employed. Preliminary trial was carried on mobile phase containing $10 \mathrm{mM}$ potassium dihydrogen phosphate monohydrate, $\mathrm{pH}$ adjusted to 4.0 with phosphoric acid and methanol $(50: 50, v / v)$ was chosen on a C18 stationary phase with a $25 \mathrm{~cm}$ length, $4.6 \mathrm{~mm}$ ID and $5 \mu$ particle size and retention time found to be high and peak is not in good shape. The proportion of the mobile phase components was optimized to reduce retention times and enable good resolution of amlexanox from the degradation products obtained by base degradation. When $\mathrm{pH}$ increased towards basic side (pH 4.8) the retention time and the resolution between the degradants and amlexanox was improved. To further reduce the retention time, the methanol proportion was increased and the observed retention time of Amlexanox was found to be about $5.9 \mathrm{~min}$.

Under optimized concentration of $10 \mathrm{mM}$ Potassium dihydrogen phosphate monohydrate, $\mathrm{pH}$ adjusted to 4.8 with phosphoric acid and methanol $(30: 70 \mathrm{v} / \mathrm{v})$ as mobile phase, the typical retention times of degradants in basic condition and amlexanox were found to be about 4.2, 4.9, 7.2 for degradants and 5.9 for amlexanox respectively (Figure 2). 


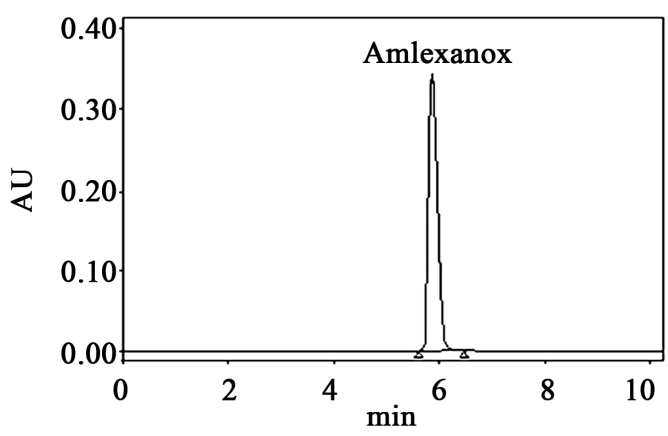

(a)

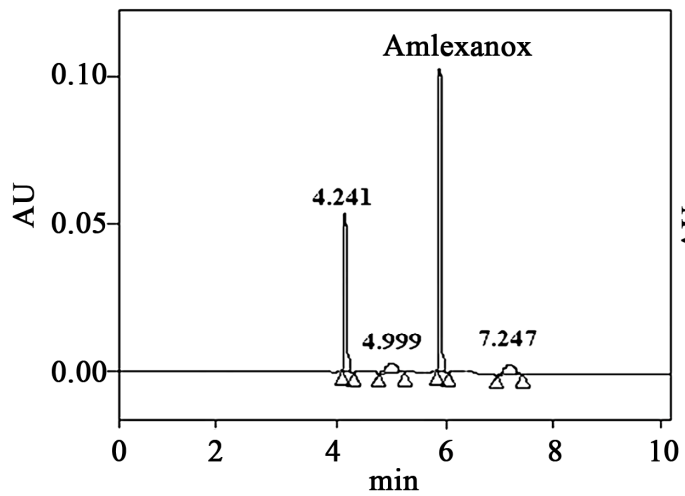

(c)

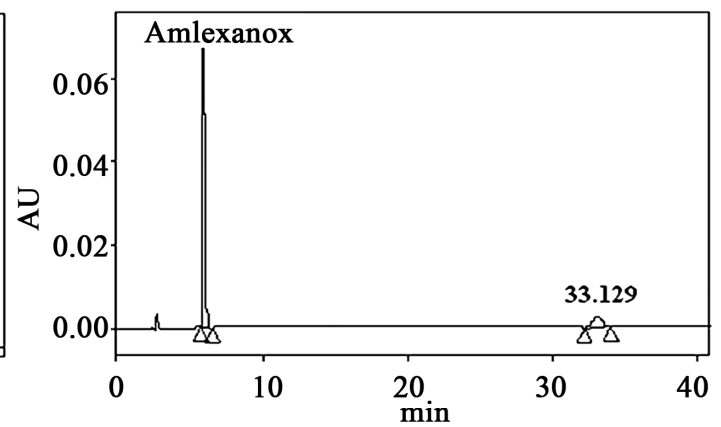

(b)

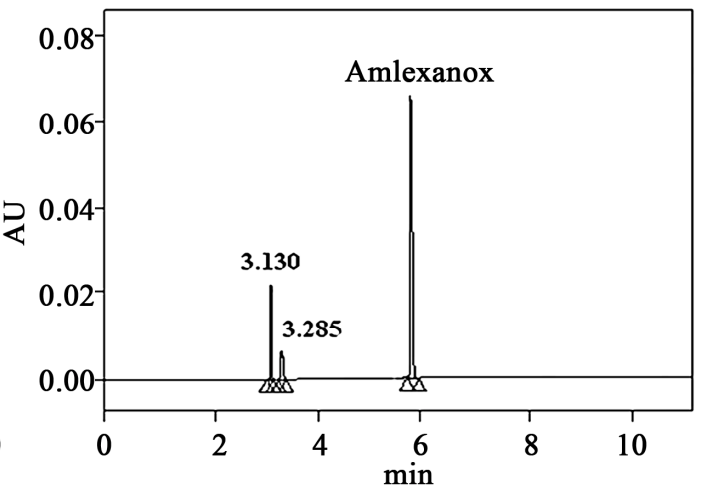

(d)

Figure 2. Typical chromatograms of (a) Amlexanox sample, (b) acid stress, (c) base stress and (d) UV exposed stress samples.

Buffer $\mathrm{pH}$ and percentage of methanol played a key role in achieving the good separation between the degradants and amlexanox besides enhancing chromatographic efficiency. The system suitability results were given in [Table 1].

\subsection{Results of Forced Degradation Studies}

The drug was exposed to $0.1 \mathrm{~N}$ methanolic $\mathrm{NaOH}$ refluxed at $100^{\circ} \mathrm{C}$ temperature for $1 \mathrm{hr}$. Amlexanox has shown significant sensitivity towards the treatment with $0.1 \mathrm{~N} \mathrm{NaOH}$ leading to observed degradation of about $12 \%$.

The drug was exposed to $1 \mathrm{~N}$ methanolic $\mathrm{HCl}$ refluxed at $60^{\circ} \mathrm{C}$ for $3 \mathrm{hrs}$ caused significant degradation (8.3\%). UV Light exposed solution caused significant degradation $\left(6.58 \%\right.$ degraded at UV $\left.200 \mathrm{~W} / \mathrm{hm}^{2}\right)$.

No major degradation products were observed when the sample was stressed in an oxidative condition (5\% methanolic $\mathrm{H}_{2} \mathrm{O}_{2}$, heated at $60^{\circ} \mathrm{C}$ for $7 \mathrm{hrs}$ ), neutral, fluorescent and thermal conditions.

From the degradation studies, Peak purity test results derived from PDA detector, confirmed that the amlexanox peak was homogeneous and pure in all the analyzed stress samples. The mass balance of stressed samples was close to $99.97 \%$. No degradants were observed after 45 minutes in the extended runtime of 90 minutes of all the amlexanox samples. The developed LC method was found to be specific in the presence of its degradation products confirm the stability indicating power of the developed method.

\subsection{Method Validation}

\subsubsection{Precision}

The \%RSD of amlexanox during precision study and intermediate precision study was $0.1 \%$ and confirming the good precision of the developed analytical method.

\subsubsection{Linearity and Range}

Linear calibration plot for assay method was obtained over the calibration ranges tested, i.e. $48-72 \mu \mathrm{g} \mathrm{m} \mathrm{L}^{-1}$ and the correlation coefficient obtained was greater than 0.999. The result shows an excellent correlation existed between the peak area and concentration of the analyte.

Table 1. System suitability data.

\begin{tabular}{ccccc}
\hline Name & $\begin{array}{c}\text { Retention } \\
\text { time }\left(t_{r}\right) \text { in } \\
\text { min }\end{array}$ & $\begin{array}{c}\text { USP } \\
\text { Resolution } \\
\left(R_{s}\right)\end{array}$ & $\begin{array}{c}\text { USP } \\
(T)\end{array}$ & $\begin{array}{c}\text { Theoretical } \\
\text { plates } \\
(N)\end{array}$ \\
\hline $\begin{array}{c}\text { Closest eluted } \\
\text { degradant } \\
\text { Amlexanox }\end{array}$ & 4.9 & - & 0.99 & 6363 \\
\hline
\end{tabular}


The best-fit linear equation obtained was $\mathrm{Y}=36422 x$ +338053 . At all concentration levels, standard deviation of peak area was significantly low and RSD was below $1.0 \%$. Analysis of residuals indicated that residuals were scattered within $\pm 2.0 \%$ with respect to $100 \%$ concentration response.

\subsubsection{Accuracy}

The percentage recovery of amlexanox in bulk drug samples ranged from $98.86 \%$ - 101.05\% [Table 2].

\subsubsection{Robustness}

Close observation of analysis results for deliberately changed chromatographic conditions (flow rate, $\mathrm{pH}$ and column temperature) revealed that the resolution between closely eluting degradant in basic condition and amlexanox was always greater than 2.5, illustrating the robustness of the method [Table 3].

\subsubsection{Solution Stability and Mobile Phase Stability}

The \%RSD of assay of amlexanox during solution stability and mobile phase stability experiments was within $1.0 \%$ RSD. No significant changes were observed in the content of amlexanox during solution stability and mobile phase stability experiments. The solution stability and mobile phase stability experiments data confirms that sample solutions and mobile phase used during assay and related substance determination were stable up to the study.

\subsubsection{Assay Analysis}

Analysis was performed for different batches of amlexanox in bulk drug samples $(n=3)$ ranged from $99.5 \%$ $100.2 \%$.

Table 2. Results of Accuracy study for Bulk drugs.

\begin{tabular}{ccc}
\hline $\begin{array}{c}\text { Added }(\boldsymbol{\mu g}) \\
(\boldsymbol{n}=\mathbf{3})\end{array}$ & $\begin{array}{c}\text { \%Recovery for } \\
\text { Bulk drugs }\end{array}$ & $\begin{array}{c}\text { \%RSD for } \\
\text { Bulk drugs }\end{array}$ \\
\hline 48 & 101.05 & 0.23 \\
60 & 100.27 & 0.39 \\
72 & 98.86 & 0.04 \\
\hline
\end{tabular}

$n=3$, Number of determinations

Table 3. Results of robustness study.

\begin{tabular}{|c|c|c|c|}
\hline S. No & Parameter & Variation & $\begin{array}{c}\text { Resolution }\left(R_{s}\right) \\
\text { between base degradant } \\
\text { and Amlexanox }\end{array}$ \\
\hline 1 & Temperature & $\begin{array}{l}\text { (a) At } 20^{\circ} \mathrm{C} \\
\text { (b) At } 30^{\circ} \mathrm{C}\end{array}$ & $\begin{array}{l}2.8 \\
2.9\end{array}$ \\
\hline 2 & Flow rate & $\begin{array}{l}\text { (a) At } 0.8 \mathrm{~mL} \cdot \mathrm{min}^{-1} \\
\text { (b) At } 1.2 \mathrm{~mL} \cdot \mathrm{min}^{-1}\end{array}$ & $\begin{array}{l}2.9 \\
2.7\end{array}$ \\
\hline 3 & $\mathrm{pH}$ & $\begin{array}{l}\text { (a) At } 4.7 \\
\text { (b) At } 4.9\end{array}$ & $\begin{array}{l}2.8 \\
2.7\end{array}$ \\
\hline
\end{tabular}

\section{Conclusions}

The Stability Indicating RP-LC method developed for quantitative determination of amlexanox in bulk drugs is precise, accurate and specific. The method was completely validated showing satisfactory data for all the method validation parameters tested. The developed method is stability indicating and can be used for the routine analysis of production samples and also to check the stability of amlexanox sample.

\section{References}

[1] J. Bell, "Amlexanox for the Treatment of Recurrent Aphthous Ulcers,” Clinical Drug Investigation, Vol. 25, No. 9, 2005, pp. 555-566. doi:org/10.2165/00044011-200525090-00001

[2] H. Makino, T. Saijo, Y. Ashida, H. Kuriki and Y. Maki, "Mechanism of Action of an Antiallergic Agent, Amlexanox (AA-673), in Inhibiting Histamine Release from Mast Cells," International Archives of Allergy and Immunology, Vol. 82, No. 1, 1987, pp. 66-71. doi:org/10.1159/000234292

[3] C. Scully and S. Porter, "Oral mucosal disease: Recurrent aphthous stomatitis,” British Journal of Oral and Maxillofacial Surgery, Vol. 46, No. 3, 2008, pp. 198-206. doi:org/10.1016/j.bjoms.2007.07.201

[4] J. Liu, X. Zeng, Q. Chen, Y. Cai, F. Chen, Y. Wang, H. Zhou, M. Lin, J. Shi, Z. Wang and Y. Zhang, "An Evaluation on the Efficacy and Safety of Amlexanox Oral Adhesive Tablets in the Treatment of Recurrent Minor Aphthous Ulceration in a Chinese Cohort: A Randomized, Double-Blind, Vehicle-Controlled, Unparallel Multicenter Clinical Trial,” Oral Surgery Oral Medicine Oral Pa-Thologz Oral Radiology and Endodontology, Vol. 102, No. 4, 2006, pp. 475-481.

[5] M. Sugiura, R. Hayakawa and T. Osada, "Fixed drug Eruption Due to Amlexanox," Contact Dermatitis, Vol. 38, No. 2, 1998, pp. 65-67. doi:org/10.1111/j.1600-0536.1998.tb05654.x

[6] S. McComish, A. Mize, J. Harris, N. Premkumar and L. E. Colon, “A High-Pressure Liquid Chromatographic Method for Measuring Mitotane: [1,1-(o,p-Dichlorodiphenyl)-2,2Dichloroethane] and Its Metabolite 1,1-(o,p'-Dichlorodiphenyl)-2,2-dichloroethene in Plasma," Therapeutic Drug Monitoring, Vol. 17, No. 5, 1995, pp. 526-531. doi:org/10.1097/00007691-199510000-00015

[7] T Shishibori, "Three Distinct Anti-Allergic Drugs, Amlexanox, Cromolyn and Tranilast, Bind to S100A12 and S100A13 of the S100 Protein Family," Biochemical Journal, Vol. 338, 1999, pp. 583-589.

[8] A. Khandwala, R. G. V. Inwegen and M. C. Alfano, “5\% Amlexanox Oral Paste, a New Treatment for Recurrent Minor Aphthous Ulcers: I. Clinical Demonstration of Acceleration of Healing and Resolution of Pain,” Oral Surgery Oral Medicine Oral Pathologz Oral Radiology and Endodontology, Vol. 83, No. 2, 1997, pp. 222- 230. 
[9] A. Khandwala, R. G. V. Inwegen, M. R. Charney and M. C. Alfano, "5\% Amlexanox Oral Paste, a New Treatment for Recurrent Minor Aphthous Ulcers II: Pharmacokinetics and Demonstration of Clinical Safety," Oral Surgery Oral Medicine Oral Pathologz Oral Radiology and Endodon- tology, Vol. 83, No. 2, 1997, pp. 231-238.

[10] J. Jacob, "Stable Viscous Liquid Formulations of Amlexanox for the Prevention and Treatment of Mucosal Diseases and Disorders,” US Patent App. 09/971, 2001, p. 562.

[11] USP, “United States pharmacopoeia," 32nd Edition, United States Pharmacopeial Convention, Rockville, 2008.
[12] International Federation of Pharmaceutical Manufactures \& Associations, "ICH Stability Testing of New Drug Substances and Products Q1A (R2)," International Conference on Harmonization, Geneva, 2003.

[13] M. Bakshi and S. Singh, "Development of Validated Stability-Indicating Assay Methods-Critical Review," Journal of Pharmaceutical and Biomedical Analysis, Vol. 28, No. 6, 2002, pp. 1011-1040. doi:org/10.1016/S0731-7085(02)00047-X

[14] U. S. Food and Drug Administration, "ICH Guidelines on Validation of Analytical Procedures: Text and Methodology Q2 $\left(\mathrm{R}_{1}\right)$,” Federal Register, FDA, Silver Spring 2005. 